\title{
Análisis de las irreversibilidades en un proceso de paro y arranque en un sistema de refrigeración con $\mathbf{R}-134 a$
}

\section{Analysis of irreversibility's in a stop and start process in a refrigeration system with R-134a}

RANGEL-ROMERO, Carlos $\dagger^{*}$, ROJAS-GARNICA, Juan Carlos, FLORES-MARTÍNEZ, Guillermo y BARCELATA-PINZÓN, Antonio

Universidad Tecnológica de Puebla, México.

ID $1^{\text {er }}$ Autor: Carlos, Rangel-Romero / ORC ID: 0000-0003-4879-4228, CVU CONACYT ID: 894477

ID $1^{\text {er }}$ Coautor: Juan Carlos, Rojas-Garnica / ORC ID: 0000-0002-2261-587X, CVU CONACYT ID: 66417

ID $2^{\text {do }}$ Coautor: Guillermo, Flores-Martínez / CVU CONACYT ID: 169853

ID $3^{\text {er }}$ Coautor: Antonio, Barcelata-Pinzón / CVU CONACYT ID: 160211

DOI: $10.35429 / J S I .2020 .15 .4 .1 .6$

Recibido Julio 05, 2020; Aceptado Diciembre 30, 2020

\section{Resumen}

En este trabajo se hace una evaluación de la energía en un proceso de arranque-paro mediante el análisis de las irreversibilidades generadas en un sistema de refrigeración por compresión mecánica de vapor con refrigerante R134a a un flujo de $1.0 \mathrm{~L} / \mathrm{s}$. Este sistema se encuentra instalado en el LABINTHAP de la SEPI-ESIME-IPN. Para este análisis se cuenta con un software que captura los datos de las presiones y temperaturas del refrigerante a la entrada y salida del evaporador, compresor, condensador y válvula de expansión en intervalos de un minuto. Para el análisis de las irreversibilidades generadas, se emplearon las leyes primera y segunda de la termodinámica. $\mathrm{Y}$, en el proceso de evaluación de paro y arranque, se demostró que el compresor marca una tendencia de un mayor consumo de energía por lo que se propone un proceso de regulación del sistema de refrigeración por compresión mecánica de vapor.

COP, Ciclo real, Diagrama T-s, Entropía, Entalpía

\begin{abstract}
In this work, an evaluation of the energy in a start-stop process is made by analyzing the generated irreversibilities in a refrigeration system by mechanical vapor compression with R-134a refrigerant at a flow of 1.0 $\mathrm{L} / \mathrm{s}$. This system is installed in the LABINTHAP of the SEPI-ESIME-IPN. For this analysis, there is software that captures data on the pressures and temperatures from the refrigerant at the inlet and outlet of the evaporator, compressor, condenser, and expansion valve at oneminute intervals. For the analysis of the generated irreversibilities, the first and second laws of thermodynamics were used. And, in the process evaluation of stopping and starting, it was shown that the compressor sets a trend of higher energy consumption, so a process of regulation of the refrigeration system by mechanical compression of steam is proposed.
\end{abstract}

COP, real Cycle, T-s Diagram, Entropy, Enthalpy

Citación: RANGEL-ROMERO, Carlos, ROJAS-GARNICA, Juan Carlos, FLORES-MARTÍNEZ, Guillermo y BARCELATA-PINZÓN, Antonio. Análisis de las irreversibilidades en un proceso de paro y arranque en un sistema de refrigeración con R-134a. Revista de Innovación Sistemática. 2020. 4-15:1-6.

*Correspondencia al Autor (Correo Electrónico: carlos.rangel@utpuebla.edu.mx)

$\dagger$ Investigador contribuyendo como primer autor. 


\section{Introducción}

Los sistemas de refrigeración por compresión mecánica de vapor son esenciales en la vida diaria del ser humano y tienen gran aplicación en los sectores industrial, comercial, doméstico y en el acondicionamiento del aire. Los equipos de refrigeración establecen un flujo de energía entre el medio que se quiere enfriar y el medio ambiente que actúa como sumidero térmico, esto se logra por medio del suministro de energía, que para este caso se hace en forma de trabajo mecánico. En particular, estos sistemas de refrigeración se deben mantener operando eficientemente para brindar las temperaturas requeridas del espacio a enfriar, pero, cuando existe algún problema en el compresor, repercute directamente en la temperatura del espacio a enfriar, por ser indispensable en el sistema, es por ello por lo que se deben mantener las características de funcionamiento apropiadas.

En el presente trabajo se describe el análisis del uso de la energía que se presenta en un paro $y$ arranque en un sistema de refrigeración por compresión mecánica de vapor, con ello se pretende mejorar el desempeño del compresor $y$ asi tener un funcionamiento apropiado en el evaporador, condensador y vávula de expansión. Tambien con el análisis de la energía en el proceso de paro y arranque de los sistemas de refrigeración se obtendrá una mayor vida útil y desempeño en el compresor a partir de una mejor estabilización de la energía y funcionamiento apropiado.

Este sistema de refrigeración ha sido estudiado exhaustivamente desde el punto de vista de conversión de la energía, a través de la primera ley de la termodinámica, además, se ha puesto atención al uso eficiente de la energía, debido a los problemas que se tienen con el suministro y el precio de esta. El buen o mal uso de la energía solo puede ser evaluado por la segunda ley de la termodinámica que permite calcular las pérdidas de energía que ocurren, como consecuencia de las irreversibilidades que se presentan en los sistemas de refrigeración.
Se presentan los resultados experimentales del uso de la energía en un proceso de paro y arranque en un sistema de refrigeración por compresión mecánica de vapor empleando como fluido de trabajo al R-134a; considerando que darlos a conocer pueda despertar el interés en la comunidad por este tema tan importante que es el uso eficiente de la energía.

\section{Sistema de refrigeración experimental por compresión mecánica de vapor}

El sistema de refrigeración que se usó para realizar este trabajo se muestra en forma esquemática en la figura 1 .

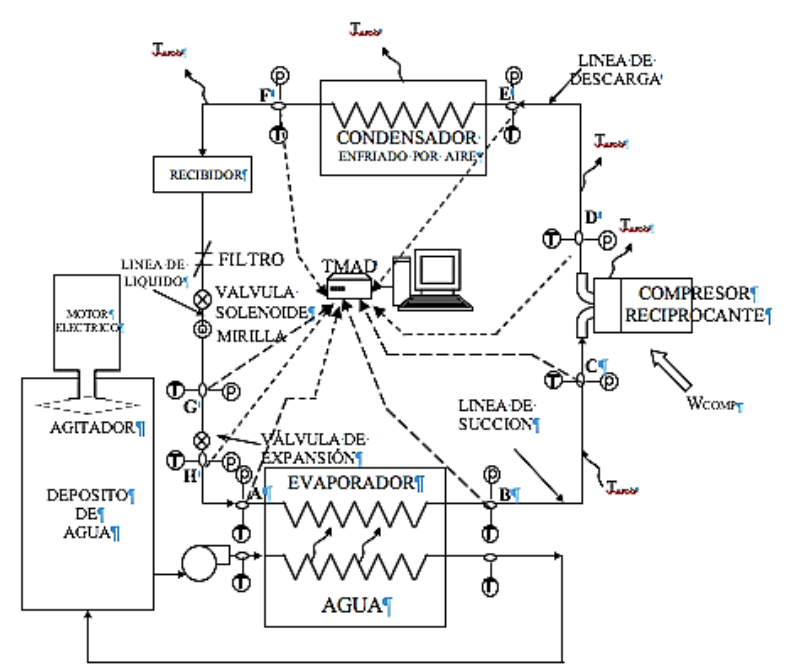

Figura 1 Componentes principales de un sistema de refrigeración por compresión mecánica de vapor Fuente: Elaboración propia

Como se muestra en la figura 1, el agua del depósito a enfriar, circula a través del evaporador, en donde el refrigerante absorbe el calor del agua, disminuyendo así la temperatura del agua, para este trabajo, con ambos refrigerantes, el flujo de masa de agua que se hace circular por el evaporador es de $1.0 \mathrm{~L} / \mathrm{s}$, posteriormente se toman lecturas de temperatura y presión del refrigerante mediante termopares y transductores de presión colocados en las entradas y salidas del evaporador, del compresor, y de la válvula de expansión. Cada prueba tiene una duración de 2 horas. 
Por otro lado para realizar la mulación de paro y arranque, el equipo de refrigeración se realiza un paro cada 50 minutos y antes de realizar el arranque se le suministra al depósito de agua a enfriar un calentamiento mediante un serpentín con la finalidad de tener la misma condición que se tuvo al inicio de la experimentación que es de 20.5 C y así observar el comportamiento de la energía mediante un proceso de paro y arranque, este comportamiento se realiza mediante las irreversibilidades generadas en el evaporador, compresor, condensador y válvula de expansión.

\section{Método de evaluación del proceso de paro y arranque mediante las irreversibilidades generadas en el sistema de refrigeración}

El ciclo termodinámico de un sistema de refrigeración por compresión mecánica de vapor se muestra en la Fig. 2 y está compuesto de los siguientes procesos: I) evaporación (4-1): el refrigerante absorbe calor del espacio a enfriar; II) absorción de calor en la tubería de succión (1$\left.1^{\prime}\right)$; III) compresión (1'-2): se incrementa la temperatura y presión del refrigerante; IV) rechazo de calor en la tubería de descarga (2-2'); V) condensación (2'-3): el refrigerante rechaza calor al medio ambiente; $\mathrm{VI}$ ) rechazo de calor en la tubería de líquido (3-3'); VII) expansión (3’4): el refrigerante sufre una caída súbita de presión.

En la Figura 2 se muestra con el diagrama P-h la comparación entre el ciclo real e ideal de refrigeración por compresión mecánica de vapor. Se observa que la diferencia entre ambos ciclos se debe a las caídas de presión, sobrecalentamiento, subenfriamiento, transferencia de calor, lo que ocasiona que las irreversibilidades se generen en el sistema de refrigeración.

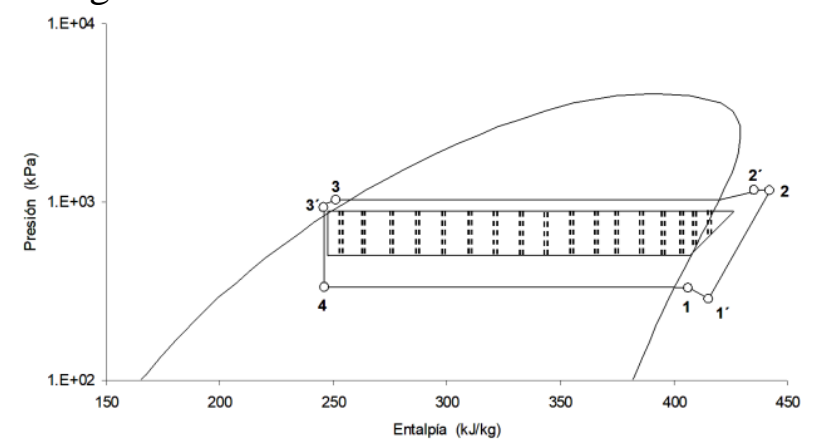

Figura 2 Comparación del ciclo de refrigeración real e ideal

Fuente: Elaboración propia

\begin{tabular}{|r|l|l|}
\multicolumn{2}{|c|}{$1^{\text {a }}$ Ley } & \multicolumn{2}{c|}{$2^{\text {a Ley }}$} \\
r & \multicolumn{1}{|c|}{$I_{4-1}=\left[m_{r}\left(s_{1}-s_{4}\right)-\frac{Q}{T_{r}}\right] T_{a m b}$} \\
\hline I & $Q_{4-1}=m_{r}\left(h_{1}-h_{4}\right)$ & $I_{1^{\prime}-1}=\left[m_{r}\left(s_{1^{\prime}}-s_{1}\right)-\frac{Q}{T_{0}}\right] T_{a m b}$ \\
\hline II & $Q_{1^{\prime}-1}=m_{r}\left(h_{1^{\prime}}-h_{1}\right)$ & $I_{2-1^{\prime}}=\left[m_{r}\left(s_{2}-s_{1^{\prime}}\right)-\frac{Q}{T_{0}}\right] T_{a m b}$ \\
\hline III & $Q_{2-1^{\prime}}=m_{r}\left(h_{2}-h_{1^{\prime}}\right)+W_{2-1^{\prime}}$ & $I_{2-2^{\prime}}=\left[m_{r}\left(s_{2^{\prime}}-s_{2}\right)-\frac{Q}{T_{0}}\right] T_{a m b}$ \\
\hline IV & $Q_{2-2^{\prime}}=m_{r}\left(h_{2^{\prime}}-h_{2}\right)$ & $I_{3-2^{\prime}}=\left[m_{r}\left(s_{3}-s_{2^{\prime}}\right)-\frac{Q}{T_{0}}\right] T_{a m b}$ \\
\hline V & $Q_{3-2^{\prime}}=m_{r}\left(h_{3}-h_{2^{\prime}}\right)$ & \\
\hline VI & $Q_{3^{-3^{\prime}}=m_{r}\left(h_{3^{\prime}}-h_{3}\right)}$ & $I_{3-3^{\prime}}=\left[m_{r}\left(s_{3^{\prime}}-s_{3}\right)-\frac{Q}{T_{0}}\right] T_{a m b}$ \\
\hline VII & $h_{3^{\prime}}=h_{4}$ & $I_{4-3^{\prime}}=\left[m_{r}\left(s_{4}-s_{3^{\prime}}\right)\right] T_{a m b}$ \\
\hline
\end{tabular}

Tabla 1 Ecuaciones de la primera y segunda ley de la termodinámica

Las ecuaciones usadas para el cálculo de los procesos del ciclo de refrigeración son las relacionadas con las leyes primera y segunda de la termodinámica, que se muestran en la tabla 1. Con estas ecuaciones se hace el análisis energético y el análisis de las irreversibilidades generadas en el sistema.

Para ello, se toman los valores de las variables termodinámicas de la entalpía y entropía del refrigerante en el sistema experimental, esto se obtiene con base en las mediciones de las temperaturas y las presiones a la entrada y salida de cada componente.

\section{Resultados experimentales}

En la Figura 3 se muestra el proceso de paro y arranque que se hace cada 50 minutos, durante un periodo de proceso de experimentación de dos horas a un flujo de $1.0 \mathrm{~L} / \mathrm{s}$, como puede observarse cuando se arranca el sistema de refrigeración la irreversibilidad en el compresor aumenta y la irreversibilidad del condensador disminuye, este sucede a los 55 minutos y 105 minutos, esta tendencia muestra un aumento de energía cada vez que un sistema de refrigeración tiene un paro y arranque. 


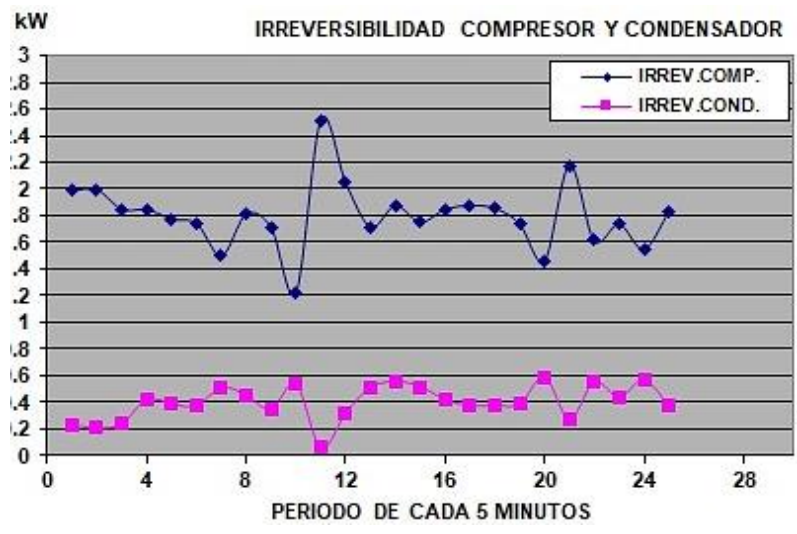

Figura 3 Comparación de irreversibilidad del compresor y condensador

Fuente: Elaboración propia

En la figura 4 se ilustra el comportamiento de las irreversibilidades generadas en el evaporador, la válvula de expansión, la tubería de succión, la tubería de descarga y la tubería de líquido, se muestra claramente cuando se realizan los procesos de paro y arranque, mostrando la misma tendencia que en el condensador, cuando el sistema de refrigeración arranca nuevamente la irreversibilidad en todos los componentes anteriormente mencionados disminuye.

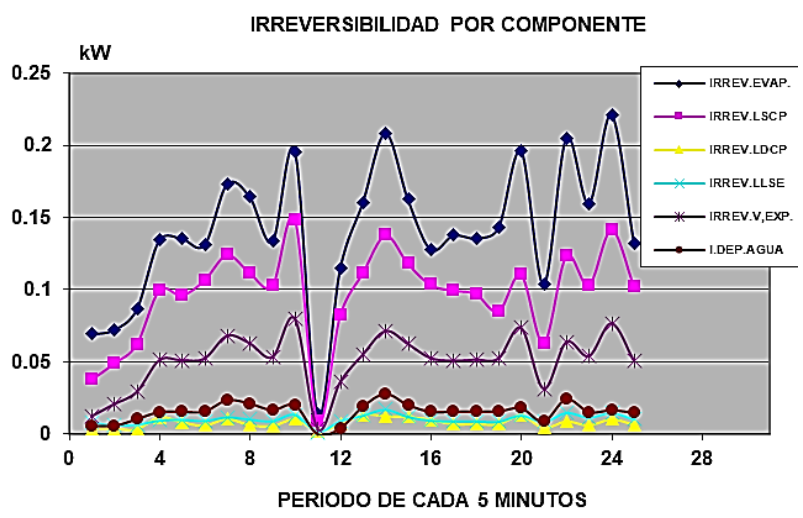

Figura 4 Comparación de la irreversibilidad por componente

Fuente: Elaboración propia

En la figura 5 se realiza la comparación entre la irreversibilidad total generada por todos los componentes, la potencia suministrada por el compresor, que presenta el mismo comportamiento que la irreversibilidad total, y la energía aprovechada.

Como se observa cuando se realiza el proceso de paro y arranque el aprovechamiento de energía tiende a disminuir, a consecuencia de que la irreversibilidad del compresor aumentó.

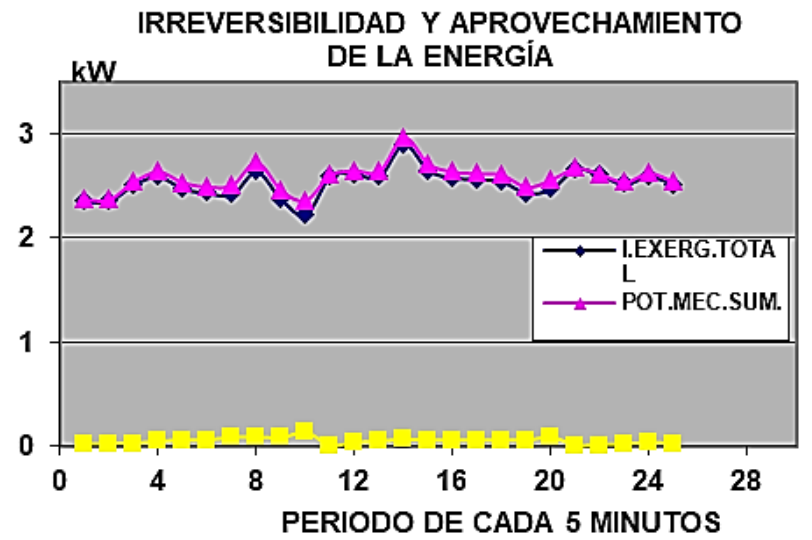

Figura 5 Comparación de irreversibilidad total contra aprovechamiento de la energía

Fuente: Elaboración propia

En la figura 6 se muestra la tendencia que tiene la temperatura del depósito de agua, que es la temperatura del espacio a enfriar con respecto a las temperaturas del refrigerante del evaporador tanto a la entrada como en la salida, y muestra una tendencia entre el evaporador y la temperatura del depósito de agua en el proceso de paro y arranque.

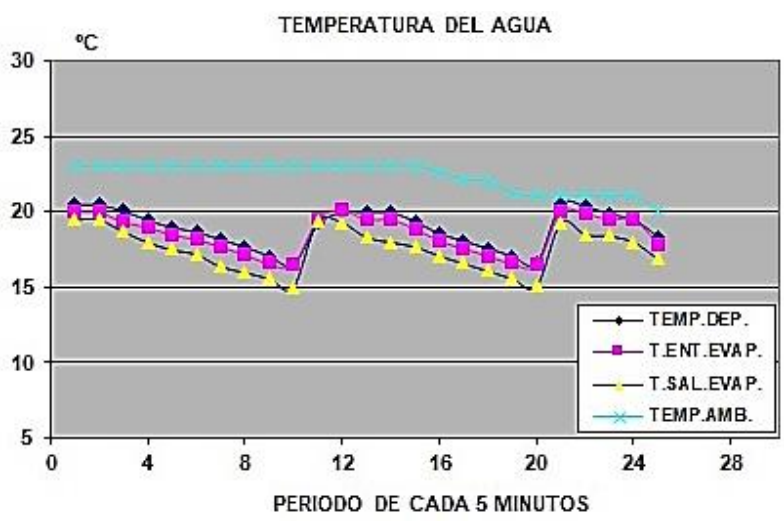

Figura 6 Comportamiento de las temperaturas Fuente: Elaboración propia

En las figuras 7 y 8, la tendencia es la misma, nos muestran que cada vez que se arranca el sistema el flujo de refrigerante y la carga térmica van en aumento.

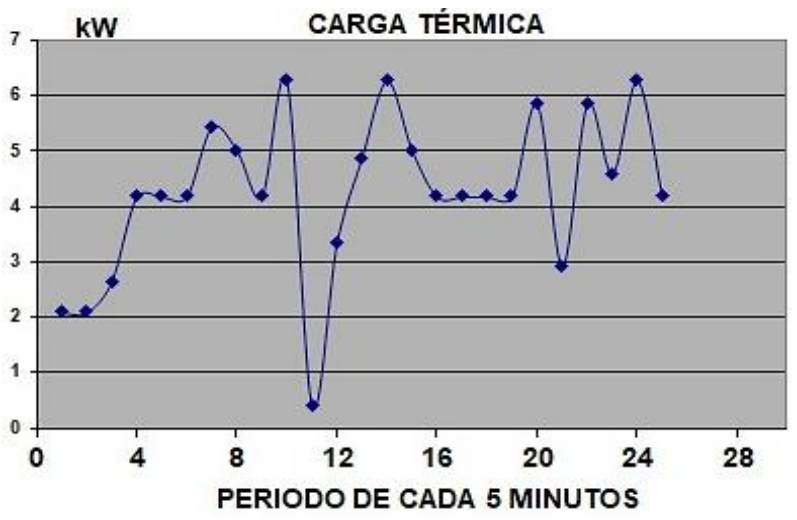

Figura 7 Comportamiento de la carga térmica Fuente: Elaboración propia

RANGEL-ROMERO, Carlos, ROJAS-GARNICA, Juan Carlos, FLORES-MARTÍNEZ, Guillermo y BARCELATA-PINZÓN, Antonio. Análisis de las irreversibilidades en un proceso de paro y arranque en un sistema de refrigeración con R-134a. Revista de Innovación Sistemática. 2020 


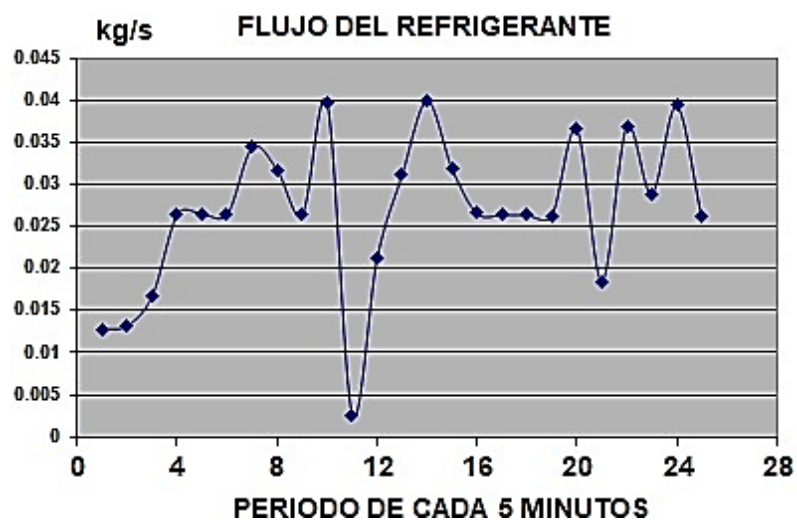

Figura 8 Comportamiento del flujo de refrigerante Fuente: Elaboración propia

\section{Análisis de resultados experimentales}

Con base a los datos experimentales se observa que los sistemas de refrigeración requieren niveles fijos de presión de descarga y presión de succión en el compresor, para no tener un mayor aumento en el consumo de energía, en contraste en el condensador está relacionada con las condiciones ambientales, esto se puede observar cuando la temperatura ambiente disminuye ya que también se reduce la presión del condensador, esto es, cuando la carga térmica es baja se requiere de una menor circulación de refrigerante. Es por ello que en un proceso de paro y arranque el compresor debe ser capaz de satisfacer los requerimientos de presión y flujo impuestos con la condición de no aumentar el consumo de energía. En un sistema de refrigeración los componentes están vinculados uno al otro, por lo que cuando un elemento modifica su régimen de funcionamiento a consecuencia de un proceso de paro y arranque, los otros componentes deben modificar el suyo instantáneamente, de tal manera que permita recuperar el equilibrio.

\section{Conclusiones}

Después de haber analizado el proceso de paro y arranque de un sistema de refrigeración, es importante regular el sistema ya que permitirá ubicar las vías de optimización de consumo de energía, como se observa en la figura 3 , que muestra un aumento en la irreversibilidad del compresor una vez iniciada el arranque del sistema, en donde se tendría que proponer una forma para disminuirla, probablemente manteniendo al compresor activo aún sin carga térmica.
La regulación del compresor es fundamental ya que consume el $80 \%$ de la energía eléctrica del sistema de refrigeración y esta se basa sobre la presión de succión que corresponde al flujo de refrigerante que circula sobre el evaporador y va de acuerdo con las necesidades del espacio a enfriar. Esta regulación se logra a través de la medición de la presión y de la temperatura a la salida del evaporador con ello se puede regular el sobrecalentamiento logrando asi disminuir el consumo de energía por parte del compresor todo ello en función de la carga térmica en el evaporador.

\section{Referencias}

Ahmadi P., Dincer I., Rosen M. A. (2013). Thermodynamic modeling and multi-objective evolutionary-based optimization of a new multigeneration energy system. Energy Conversion and Management, Vol. 76. pp. 282300 .

Ahmed B., Zubair S.M. (2011). Performance degradation of a vapour compression refrigeration system under fouled conditions. International Journal of Refrigeration. Vol. 34. pp. 1016-10271.

Anand S., Tyagi S.K. (2012). Exergy analysis and experimental study of a vapor compression refrigeration cycle. Journal of ThermalAnalysis and Calorimetry. Vol. 110. pp. 961-971.

Ansari N., Yadav B., Kumar J. (2012). Theoretical exergy analysis of HFO-1234yf and HFO-1234ze as an alternative replacement of HFC-134a in simple vapour compression refrigeration system. International Journal of Scientific Engineering Research. Vol. 4 (8). pp. 137-144.

Bahman A., Groll E. (2016). Second-law analysis to improve the energy efficiency of environmental control unit. $16^{\text {th }}$ International refrigeration and Air Conditioning Conference at Purdue, July 11-14, 2016.

Chandrasekharan M. (2014). Exergy analysis of vapor compression refrigeration system using $\mathrm{R} 12$ and R134a as refrigerants. International Journal of Students' Research in Technology \& Management. Vol. 2 (04). pp. 134-139. 
Fenga H., Chena L., Suna F., Wu. Ch. (2011). Heating load and COP optimisations for a universal steady flow endoreversible heat pump model. International Journal of Ambient Energy Vol. 32 (2). pp. 70-77.

Nawaz Md., MamoonMd., Ashar Md., Zafar A. (2015). Energy and exergy analysis of vapour compression refrigeration system with $\mathrm{R} 12$, R22, R134a. International Journal of Emerging Technology and Advanced Engineering Volume 5, Issue 3 March 2015. pp. 210-216.

Yadav P., Sharma A. (2015).Exergy analysis of R134a based vapour compression refrigeration tutor. Journal of Mechanical and Civil Engineering (IOSR-JMCE). National Conference on Advances in Engineering, Technology \& Management (AETM'15). pp. 73- 77.

Yataganbaba A., Kilicarslan A., KurtbasI. (2015). Exergy analysis of R1234yf and R1234ze as R134a replacements in a two evaporator vapour compression refrigeration system. International Journal of Refrigeration, 60:pp. 26-37.

Yataganbaba A., Kilicarslan A., Kurtbas I. (2015). Irreversibility analysis of a twoevaporator vapour compression refrigeration system. International Journal of Exergy. Vol. 18, No. 3. pp. 340-355. 